\title{
Evaluating the value of Amphiregulin, Phosphatase and Tensin Homologue (PTEN) and P21 Expression for Anti-EGFR Treatment in Metastatic Colorectal Carcinoma
}

\author{
Ahmed Ali Obaya ${ }^{1}$, Amrallah A Mohammed ${ }^{2 *}$, Hanaa Rashied ${ }^{3}$, Adel Mahmoud \\ Morsy $^{4}$, Gamal Osman ${ }^{4}$, Ahmed S Allam ${ }^{5}$, Ahmed M Elsayed ${ }^{6}$, Ola A Harb ${ }^{7}$, \\ Walid $\mathbf{S} \mathbf{H}^{7}$
}

\begin{abstract}
Background: Despite the significant progress in target therapy for the treatment of metastatic colorectal carcinoma (mCRC), the overall survival isn't satisfactory. Methods: We assessed the expression of Amphiregulin, PTEN, and $P 21$ in sections from 23 paraffin blocks prepared from 23 patients with left-sided mCRC using immunohistochemistry (IHC). The relationship between their level of expressions, clinicopathological parameters, response to anti-EGFR, and prognosis were analyzed. Results: High Amphiregulin, PTEN and low P21 expression levels were associated with low tumor grade ( $\mathrm{p}=0.038$ and 0.025 respectively), better response to anti-EGFR treatment $(\mathrm{p}<0.001)$, and favorable outcome \{progression-free survival (PFS) and overall survival (OS) $\}(\mathrm{p}<0.05)$. There was a direct relation between Amphiregulin and PTEN expressions (phi coefficient=+0.840), while there was an inverse relation between $P 21$ expression and both Amphiregulin (phi coefficient $=-0.840$ ) and $P T E N$ expressions (phi coefficient $=-1.000$ ), which was statistically significant $(\mathrm{P}<0.001)$. Conclusion: High Amphiregulin and PTEN expression levels and low P21 expression levels were associated with better response to anti-EGFR therapy and improved survival outcome. They might be considered predictive markers of response to anti-EGFR therapy in mCRC.
\end{abstract}

Keywords: Anti-EGFR treatment- metastatic colorectal carcinoma- amphiregulin- $P T E N$ - $P 21$-immunohistochemistry

Asian Pac J Cancer Prev, 22 (4), 1025-1034

\section{Introduction}

Colorectal carcinoma (CRC) is the fourth commonest diagnosed cancer and the second cause of cancer-related mortality in the United States (Siegel et al., 2019). Although the advances in the pathophysiology understanding of CRC, still its management is challenging and incurable. Anti-epidermal growth factor receptor (EGFR) combined with chemotherapy is now the standard of care in left side mCRC with EGFR sensitizing mutation (Khelwatty et al., 2013). The median overall survival (OS) improved 4.5 months by the addition of anti-EGFR monoclonal antibody (cetuximab) to chemotherapy (CT) protocol (Douillard et al., 2010).

However, it was found that certain patients have acquired a certain degree of drug resistance which limits its clinical efficacy (Khelwatty et al., 2017). Some studies showed that there are several mutations that occurred in many downstream effector molecules of the EGFR signaling pathway indicating that additional markers are needed to predict the response to anti-EGFR therapy (Chen et al., 2015).

Amphiregulin is one of the ligands of the EGF family that mediates the biological roles through binding to EGFR in both epithelial and mesenchymal cells (Yarden et al., 2001). Thus, Amphiregulin participates in cancer cell proliferation, migration, invasion, and angiogenesis in human carcinomas (Ma et al., 2001), so Amphiregulin was incriminated as a predictive biomarker of anti-EGFR therapy for most EGFR-driven carcinomas (Zarkavelis et al., 2017).

Phosphatase and tensin homologue $(P T E N)$ is an important cancer-suppressor gene and one of EGFR downstream cascade members. Loss of PTEN function has been reported in mCRC (Negri et al., 2010).

P21 and P27 are cyclin-dependent kinase (CDK)

\footnotetext{
${ }^{1}$ Department of Clinical Oncology \& Nuclear Medicine, Faculty of Medicine, Zagazig University, Egypt. ${ }^{2}$ Department of Medical Oncology, Faculty of Medicine, Zagazig University, Egypt. ${ }^{3}$ Department of Clinical Oncology \& Nuclear Medicine, Elmabara Hospital of Zagazig, Health Insurance Organization, Zagazig, Egypt. ${ }^{4}$ Department of General Surgery, Faculty of Medicine, Zagazig University, Egypt. ${ }^{5}$ Department of Internal Medicine, Faculty of Medicine, Zagazig University, Egypt. ${ }^{6}$ Department of Tropical Medicine, Faculty of Medicine, Zagazig University, Egypt. ${ }^{7}$ Department of Pathology, Faculty of Medicine, Zagazig University, Egypt.*For Correspondence: amrallaabdelmoneem@yahoo.com
} 
inhibitors linked with an increase in the cell number G2/M phase of the cell cycle. Anti-EGFR could induce cell cycle arrest at G2/M and at the G1 phase (Terzuoli et al., 2017).

Although there are several studies that assessed predictive markers of cetuximab therapy in patients with mCRC there is no previous study that clarified the role of Amphiregulin, PTEN, and P21 expression together.

In the present study, we aimed to assess Amphiregulin, PTEN and P21 expression expression in $\mathrm{mCRC}$ patients to correlate their levels of expression with clinicopathological criteria of the tumor and with the outcome of cetuximab sensitivity treatment.

\section{Materials and Methods}

This is a prospective study involving all patients with left side CRC who were operated at General Surgery Department and treated in Medical Oncology and Clinical Departments, Zagazig University hospitals during the period from December 2016 to December 2018. Patients who were clinically suspected to have CRC underwent full clinical examination, radiological evaluation in the form of pelvic abdominal computed tomography, and colonoscopy biopsies were taken and histopathological confirmation in Pathology Department, Faculty of Medicine, Zagazig University. All patients had received the specific post-operative protocol.

The inclusion criteria were age $\geq 18$ years old, pathological diagnosed CRC, left-sided wild RAS type with a metastatic disease either denovo or after adjuvant therapy. they started the systemic treatment with a cetuximab-based protocol. Only 23 patients were eligible. The samples were prepared, diagnosed, graded, and staged according to the eighth edition of the American Joint Committee on Cancer staging system (AJCC-8) classification and the World Health Organization (WHO) classification (Amin et al., 2017, Ueno et al., 2012). Clinical and pathological criteria have been identified by review of the patient's files. Patients were followed for 2 years from the date of metastatic disease diagnosis.

\section{Immunohistochemical staining}

IHC was performed on the 23 samples as previously explained (Hsu et al., 1981). The sections were incubated with the primary goat polyclonal antibody to Amphiregulin (Millipore, Billerica, MA, USA), primary rabbit monoclonal antibody to PTEN (Millipore, Billerica, MA, USA), primary mouse monoclonal antibody to p21 [EPR362] (ab109520) (Abcam, UK) dilution 1;200 for $30 \mathrm{~min}$, at room temperature. The bound primary antibodies were detected by adding anti-goat secondary antibodies for $30 \mathrm{~min}$, at room temperature. The sections were counterstained with Mayer's hematoxylin, and were finally mounted.

The expression of Amphiregulin was found in the cytoplasm and the membrane of tumor cells, the expression of PTEN was in the cytoplasm and nucleus of tumor cells, and the expression of $P 21$ expression was in the nucleus of tumor cells.

\section{Scoring criteria}

CRC tumor section immunostaining assessed and scored depending on the percentage of stained tumor cells assigning a score of 0 to 3 . Score $0=$ stained tumor cell $>$ $5 \%$, score $1=$ stained tumor cell $>10 \%$, score $2=$ stained tumor cell $>20 \%$, and score $3=$ stained tumor cell $>50 \%$. Also, the intensity of immunostaining scored from 0-3 based on the color intensity. Score $0=$ negative, score $1+$ $=$ weak light brown, score $2+=$ moderately brown, and score $3+=$ strong intense brown). To reach a final stain score of 0-9 we multiplied scores of the intensity and the extent and we considered 3 as a cut point above which is considered high expression and below which a low expression (Khelwatty et al., 2017). Correlations were done between the used markers, response to CetuximabFOLFIRI protocol, and survival.

Before starting the work, we obtained the institutional review board (IRB) approval with no funding support.

\section{Statistical analysis}

Percent of the included categorical variables in the study were compared through using appropriate tests whether; Pearson's Chi-square test or Fisher's exact test. The strength of resulted relationship between expressions of Amphiregulin, PTEN, and P21 were assessed by calculation of the phi coefficient considering $(+)$ sign as an indicator for the direct relationship and considering the (-) sign as an indicator for the inverse relationship. Overall Survival (OS) rate was considered as the time from CRC diagnosis to the time of patients' death or the time of most recent follow-up time (censored). ProgressionFree Survival (DFS) rate was considered as the time from starting CRC treatment to the date of its progression or to date of patients most recent follow-up time during which the patients were progression-free. Stratification and categorization of OS and PFS rates were assessed in relation to Amphiregulin, PTEN, and P21 expression and were estimated by using the method of Kaplan-Meier plot, and were compared by using the two-sided exact log-rank test. A p-value $<0.05$ was considered significant. Statistics of the current study were done by using SPSS 22.0 for windows (SPSS Inc., USA) and MedCalc windows (MedCalc Software bvba 13, Belgium).

\section{Results}

\section{Patient data}

The clinical data of the 23 patients with mCRC that were included in the study are summarized in Table 1.

The $23 \mathrm{mCRC}$ cases included into 16 (69.6\%) males and $7(30.4 \%)$ females. The 23 patients who received cetuximab were analyzed as; $2(8.7 \%)$ patients experienced a partial response (PR) and 13 patients (56.5\%) experienced a complete response (CR). So, the overall response (OAR) rate was $65.2 \%$. Four $(17.4 \%)$ patients experienced stable disease (SD) for more than eight weeks, while four (17.4\%) patients experienced a short-term progression of cancer (PD). The overall rate of disease control was $68.8 \%$. There is no significant difference between the rate of response to therapy and patients; age, sex, histopathological subtype, site, or 
number of distant metastases $(\mathrm{p}>0.05)$.

Amphiregulin, PTEN and P21 expression in relation to clinicopathological parameters; Table 2, Figures 1, 2 and 3

Levels of expression of Amphiregulin, PTEN, and $P 21$ were not correlated with age, sex, histopathological subtype, site, or number of distant metastases.

High Amphiregulin, PTEN, and low P21 expression levels were associated with a low grade of the tumor $(\mathrm{p}=0.038$ and 0.025 respectively) $(\mathrm{p}>0.05)$.

Association of Amphiregulin, PTEN and P21 with treatment outcome; Table 3, Figure 4

High Amphiregulin and PTEN levels and low P21 levels were associated with cetuximab-based regimen-responsive patients $(p<0.001)$ and better outcomes in both PFS and OS ( $\mathrm{p}<0.05)$.

Regarding the relation between their expressions, there was a direct relation between Amphiregulin expression and PTEN expression (phi coefficient $=+0.840$ ), an inverse relation between Amphiregulin expression and $P 21$ (phi coefficient $=-0.840$ ), and an inverse relation between PTEN expression and P21 expression (phi coefficient $=-1.000)(\mathrm{P}<0.001)$.

\section{Discussion}

Despite the effectiveness of cetuximab regarding the management of patients with mCRC, it showed a considerable number of those patients appear not to benefit from it due to acquired or inherited drug resistance in some patients (Zhang et al., 2018). So, we need to identify novel biomarkers that could predict the efficacy of cetuximab therapy and allow for more effective clinical use of such drug. Molecular targeted monoclonal antibody treatment is expensive, so choosing the best biomarkers which will be adequately used to target the real patient population is a must. Additionally, choosing patients who will have a large benefit from such agents will protect them from the unneeded side effects (Sahin et al., 2014). We have chosen three biomarkers and evaluated their expression in mCRC to predict cetuximab efficacy, moreover, we used an easy and cheap technique (IHC).

In the current study, patients with high expression of Amphiregulin in their tumors showed good response to the cetuximab-based regimen and improved both PFS and OS. This result was consistent with the finding of previous studies that demonstrated the association between high expression of Amphiregulin and better cetuximab efficacy (Okada et al., 2014, Ford et al., 2007). However,

Table 1. Clinicopathological Features, Amphiregulin, PTEN, P21 Expression and Outcome of Included Patients with Metastatic Colorectal Cancer (mCRC)

\begin{tabular}{|c|c|c|c|c|c|}
\hline \multirow[t]{2}{*}{ Characteristics } & \multicolumn{2}{|c|}{ All patients $(\mathrm{N}=23)$} & \multirow[t]{2}{*}{ Characteristics } & \multicolumn{2}{|c|}{ All patients $(\mathrm{N}=23)$} \\
\hline & No. & $\%$ & & No. & $\%$ \\
\hline Age & & & Amphiregulin & & \\
\hline Mean \pm SD & $64.69 \pm 7.47$ & & Low & 10 & $43.50 \%$ \\
\hline Median (Range) & $67(50-75$ & & High & 13 & $56.50 \%$ \\
\hline$\leq 65$ years & 11 & $47.80 \%$ & & & \\
\hline$>65$ years & 12 & $52.20 \%$ & & & \\
\hline Sex & & & PTEN & & \\
\hline Male & 16 & $69.60 \%$ & Low & 12 & $52.20 \%$ \\
\hline Female & 7 & $30.40 \%$ & High & 11 & $47.80 \%$ \\
\hline Histopathology & & & $\mathrm{P} 21$ & & \\
\hline Conventional & 20 & $87 \%$ & Low & 11 & $47.80 \%$ \\
\hline Mucinous & 3 & $13 \%$ & High & 12 & $52.20 \%$ \\
\hline Grade & & & Response & & \\
\hline Grade I & 3 & $13 \%$ & $\mathrm{PD}$ & 4 & $17.40 \%$ \\
\hline Grade II & 9 & $39.10 \%$ & SD & 4 & $17.40 \%$ \\
\hline Grade III & 11 & $47.80 \%$ & PR & 2 & $8.70 \%$ \\
\hline $\mathrm{T}$ & & & $\mathrm{CR}$ & 13 & $56.50 \%$ \\
\hline $\mathrm{T} 2$ & 5 & $21.70 \%$ & NR & 8 & $34.80 \%$ \\
\hline $\mathrm{T} 3$ & 7 & $30.40 \%$ & OAR & 15 & $65.20 \%$ \\
\hline $\mathrm{T} 4$ & 11 & $47.80 \%$ & & & \\
\hline Site of metastasis & & & Follow-up duration & & \\
\hline Liver only & 12 & $52.20 \%$ & Mean \pm SD & $22.04 \pm 3.78$ & \\
\hline Liver \& Other organ & 11 & $47.80 \%$ & Median (Range) & $24(12-24)$ & \\
\hline Liver metastasis & & & Outcome & & \\
\hline Single & 12 & $52.20 \%$ & Progression & 8 & $42.10 \%$ \\
\hline Multiple & 11 & $47.80 \%$ & Died & 7 & $30.40 \%$ \\
\hline
\end{tabular}



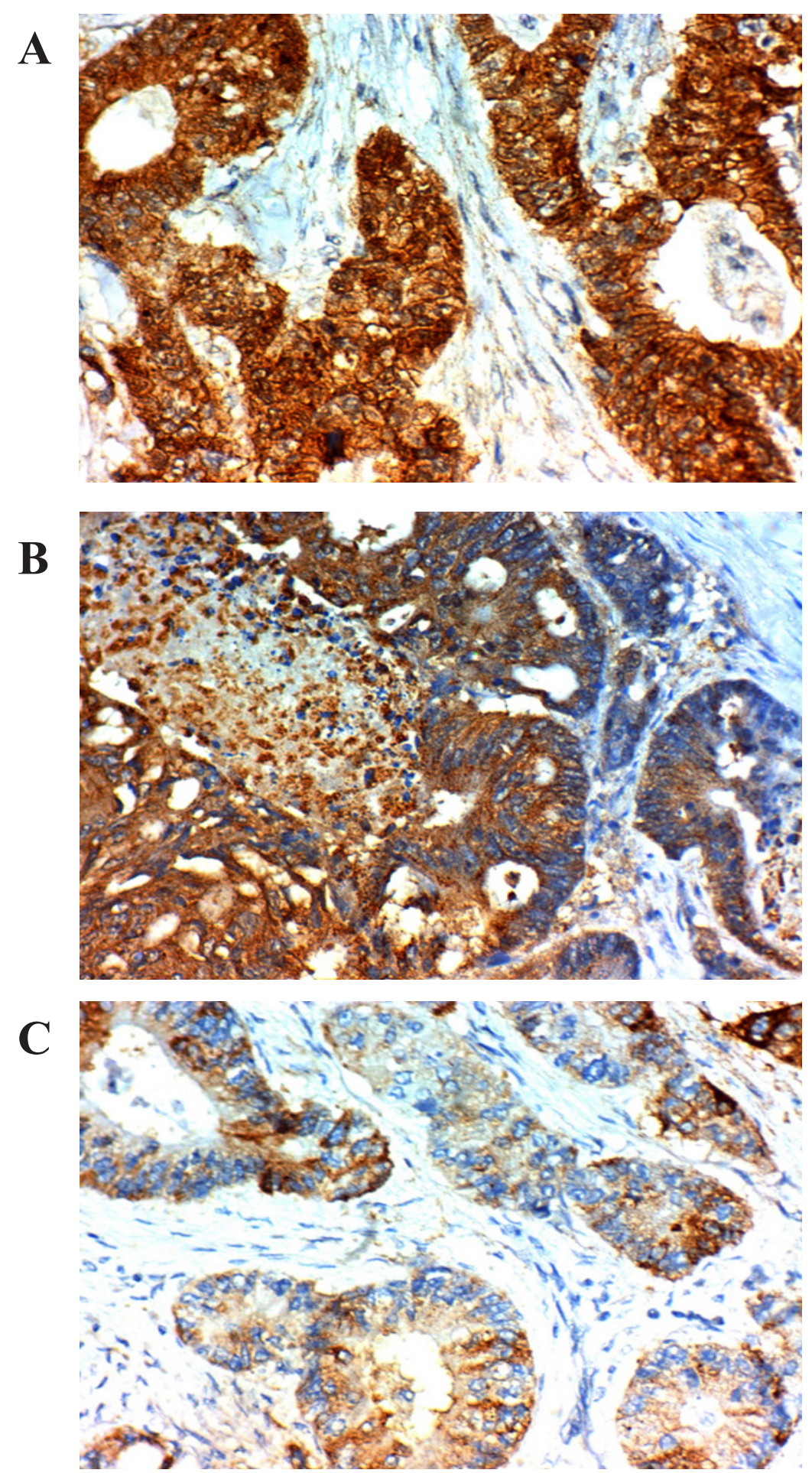

Figure 1. Amphiregulin, PTEN and P21 Expression in Relation to Clinicopathological Parameters

the methods of Amphiregulin evaluation were different; Yonesaka et al., (2015) detected the Amphiregulin level in the plasma, Jacobs et al., (2009) assessed the gene expression levels in primary tumors by grouping them according to the status of $K R A S$ mutations, Sunakawa et al., (2016) used amphiregulin $m R N A$ expression and Li et al., (2010), detected the Amphiregulin levels of expression in both tumor tissue and serum of patients with mCRC and found that Amphiregulin expression is correlated with the degree of malignant invasion, presence of distant metastasis, peri-neural and vascular invasion, which are considered unfavorable parameters for prediction of OS and PFS.

Furthermore, Ferraros et al., (2012) had proved that suppression of EGFR ligand Amphiregulin leads to cetuximab resistance which was in line with our results and explained them.

Clearly, Amphiregulin significantly affects the prognosis of mCRC patients who are treated with cetuximab-based therapy significantly; but, patients' outcomes could not be explained by a single factor (Yonesaka et al., 2015). Previous studies found that some mCRC patients abundantly expressing Amphiregulin but do not respond to cetuximab (Yonesaka et al., 2015, 


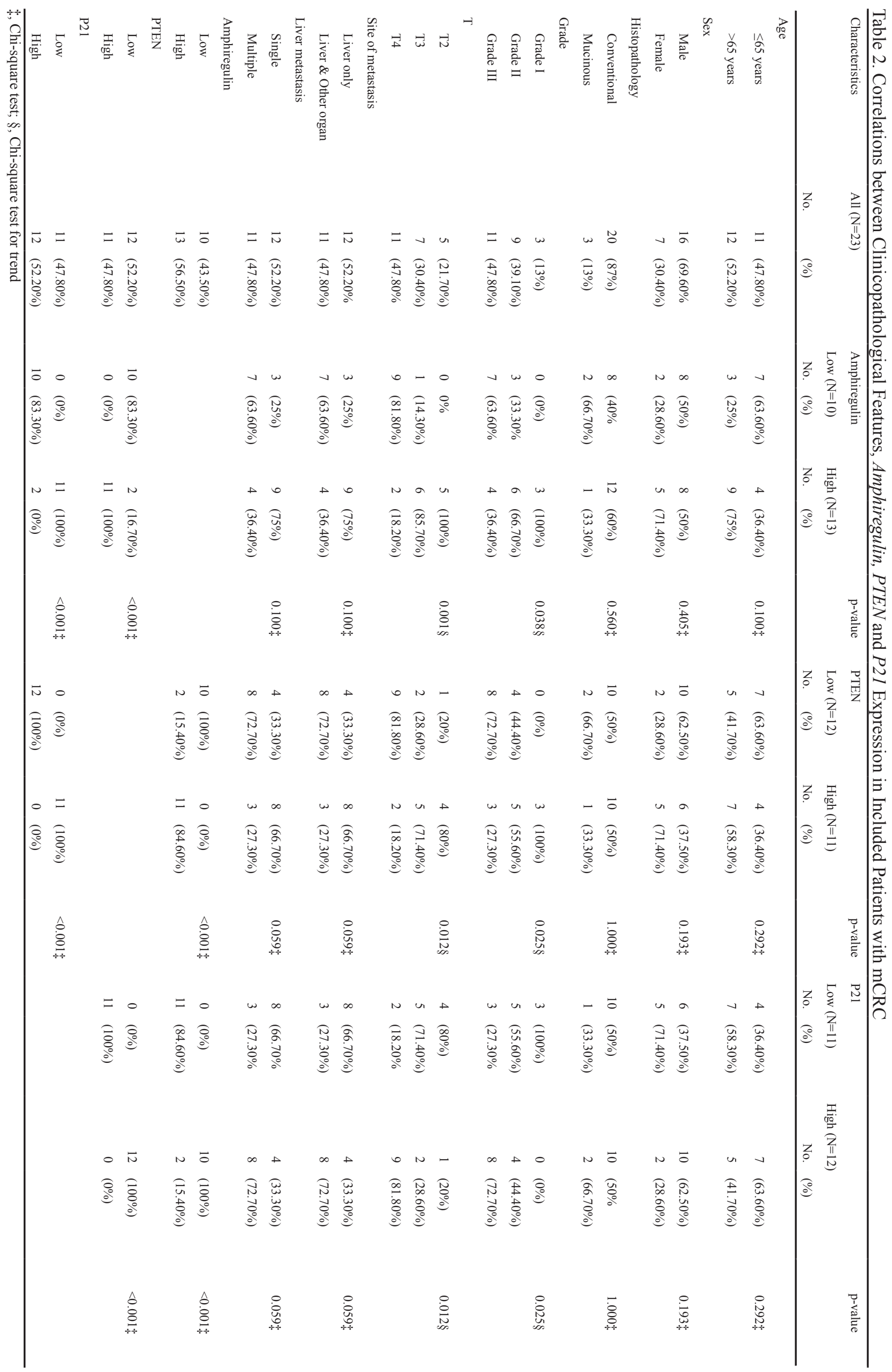



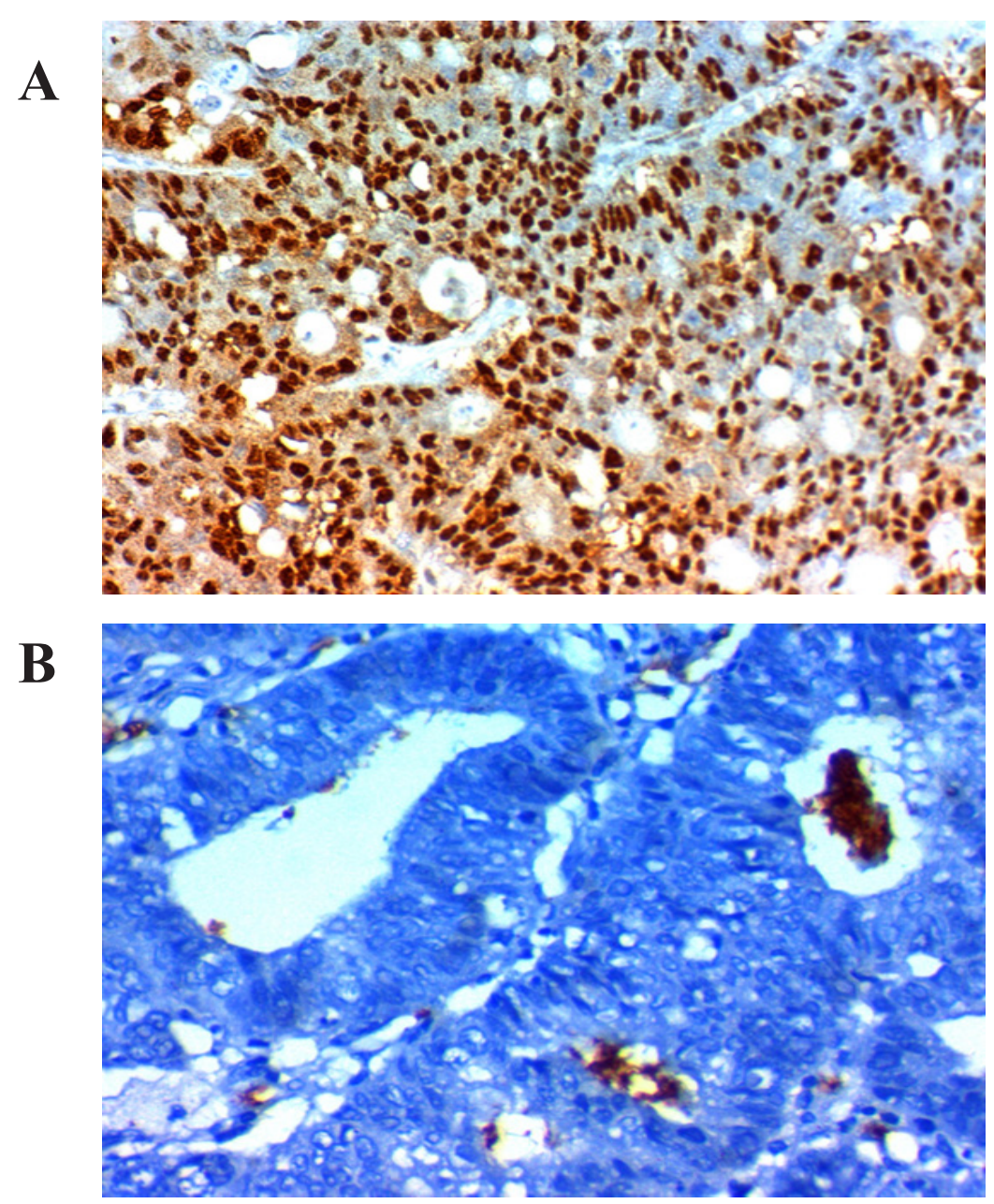

Figure 2. Amphiregulin, PTEN and P21 Expression in Relation to Clinicopathological Parameters

Ferraros et al., 2012), which could be explained by the presence of different molecules that affects cetuximab therapy. So, all ligands of EGFR should be evaluated to predict its response.

Moreover, in the present study loss of PTEN protein expression in mCRC tissues was associated with poor response to cetuximab while high levels of PTEN expression were associated with a better response. These results matched with the finding of Zhang et al., 2018 who found that PTEN deficiency predicts cetuximab resistance in $\mathrm{mCRC}$.

Also, Chen, et al., (2015) have also shown that PTEN
A

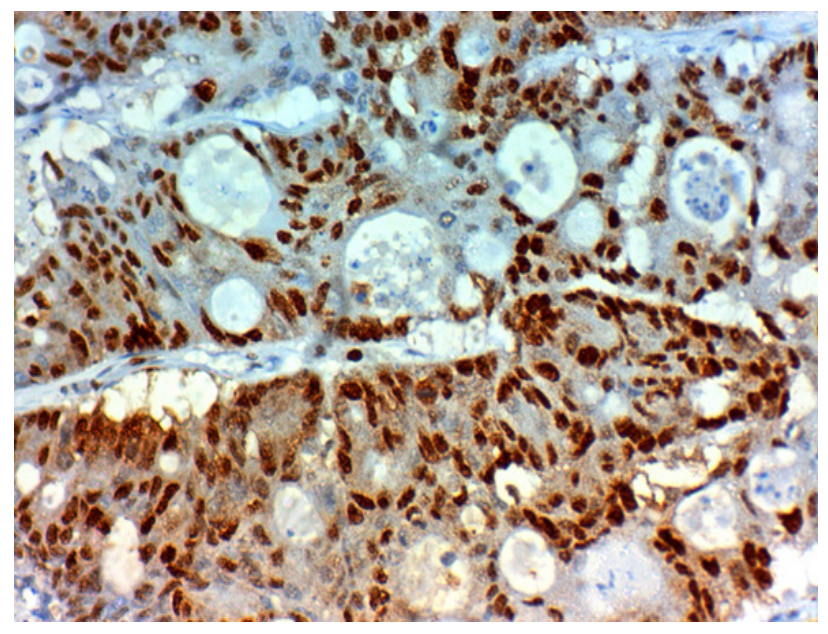

B

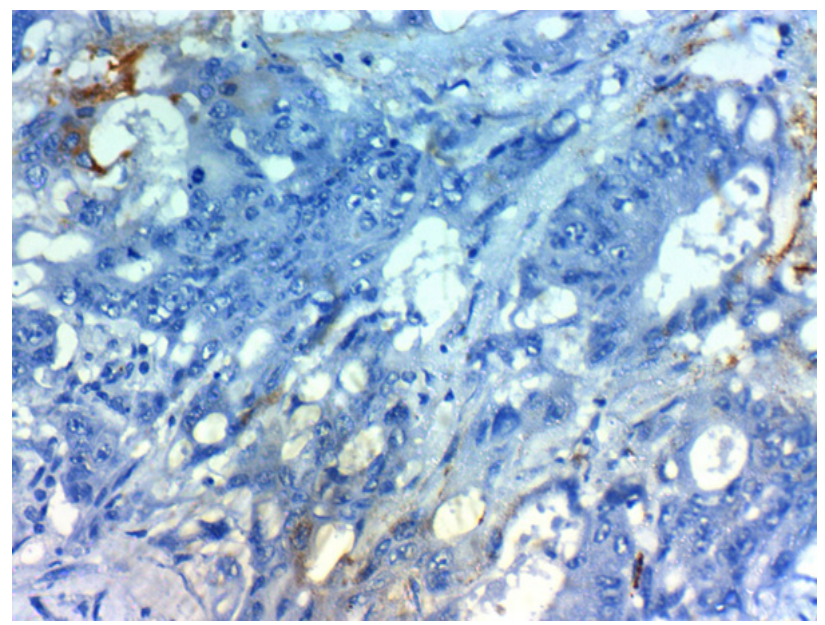

Figure 3. Amphiregulin, PTEN and P21 Expression in Relation to Clinicopathological Parameters 


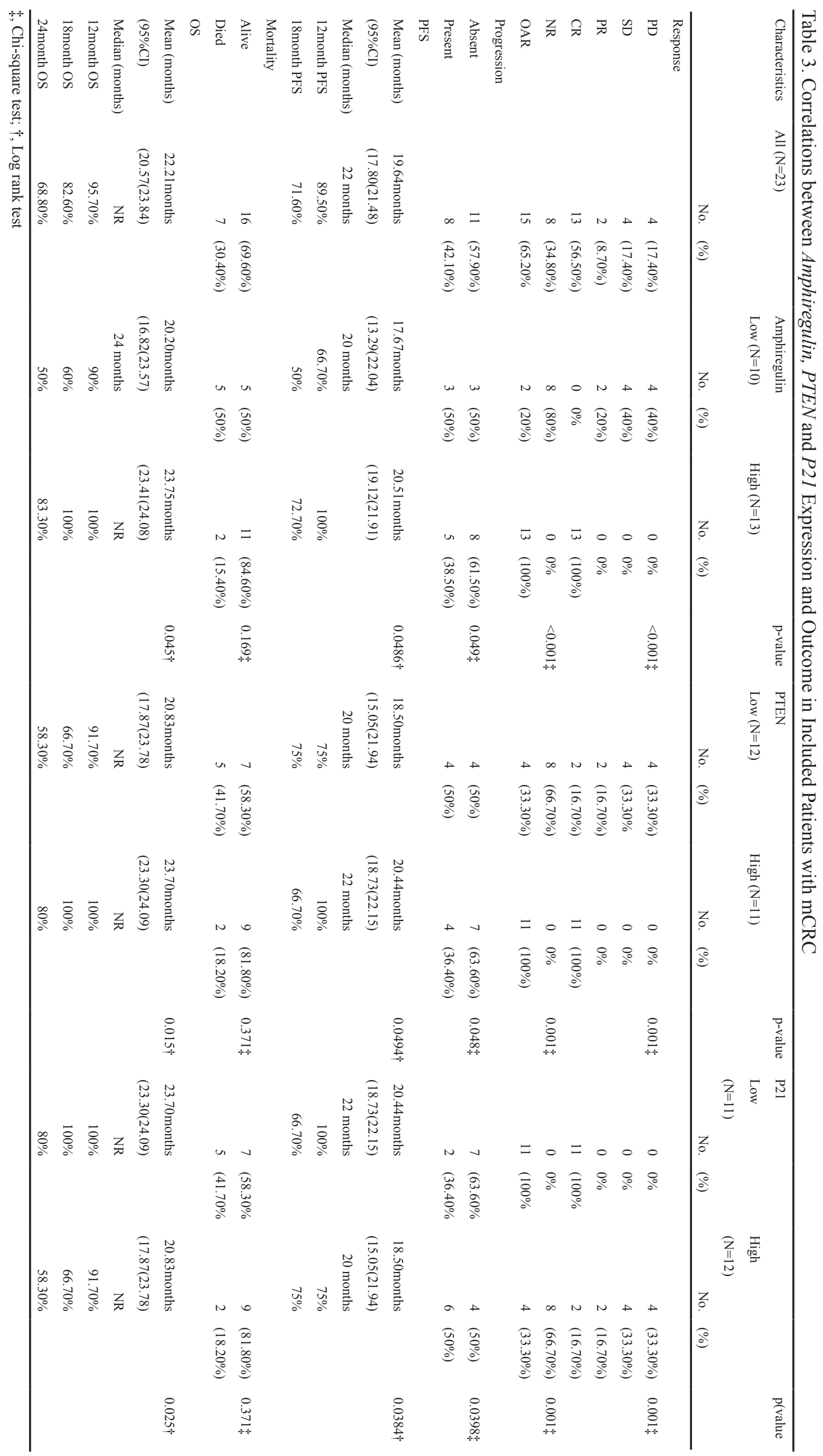



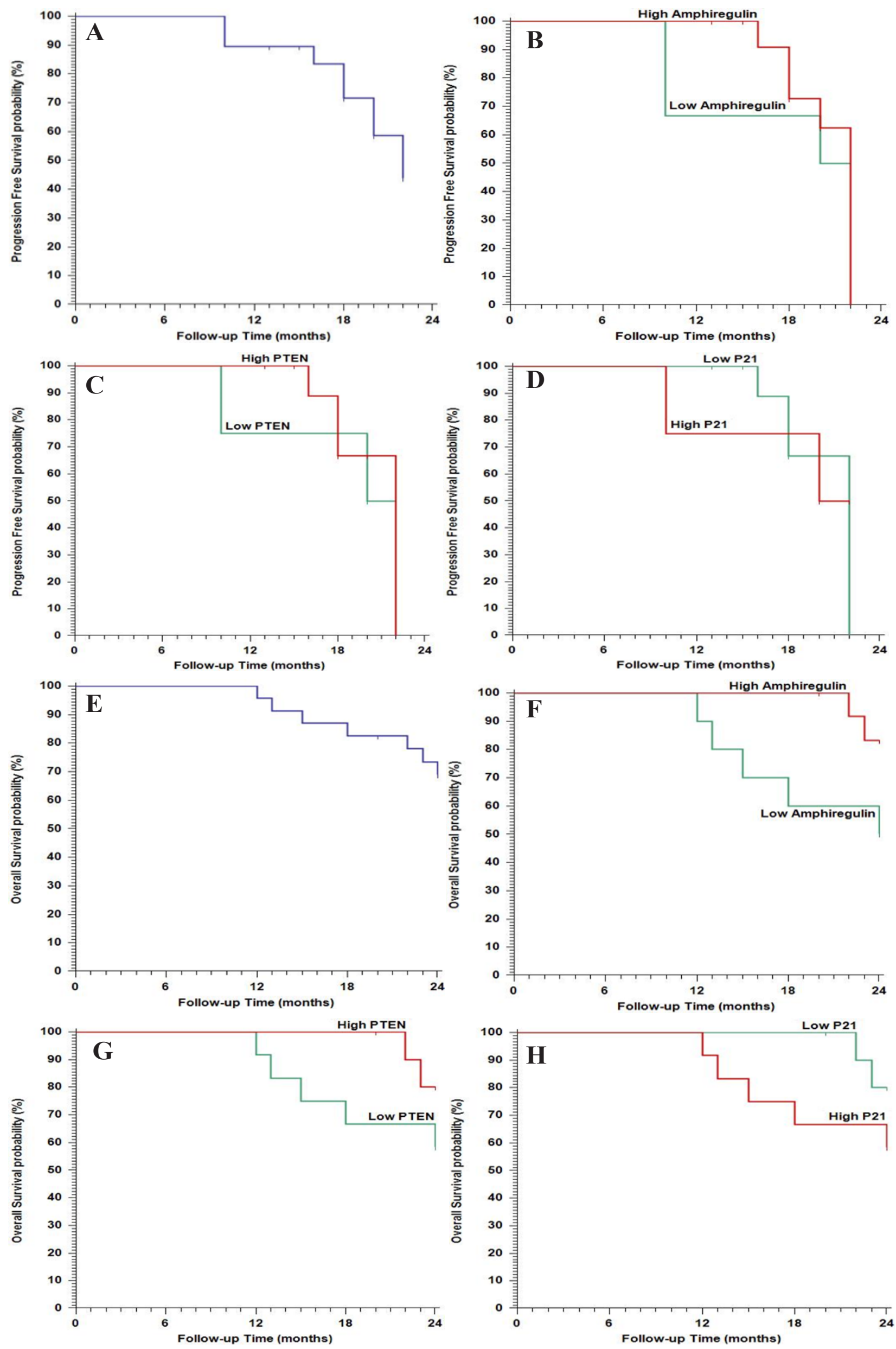

Figure 4. Association of Amphiregulin, PTEN and P21 with Treatment Outcome;

1032 Asian Pacific Journal of Cancer Prevention, Vol 22 
expression was associated with better OS rate and OAR to chemotherapy combined with cetuximab. The same findings were proved in many previous studies (Loupakis et al., 2009, Razis et al., 2008, Frattini et al., 2007, Mao et al., 2010, and Sood et al., 2012).

The mechanism of cetuximab resistance might be based on exosome-induced PTEN deletion. Tumor-derived exosomes participate in many processes, like tumor metastasis, drug resistance, and drug delivery (Shao et al., 2016, Zhang et al., 2018).

A functional interaction was detected between PTEN activity, EGFR tyrosine kinase signaling. PTEN was found to be related to EGFR-inhibitors resistance in many types of cancer (Mellinghoff et al., 2007). Such resistance is related to the negative regulator of the phosphatidylinositol 3' kinase (PI3K) complex. Inactivation of PTEN leads to uncontrolled signaling of the protein kinase $\mathrm{B}$ (PKB)/Akt pathway that in turn dissociates the inhibition of EGFR through this pathway. As wild-type PTEN is relatively common $(60-100 \%)$ in CRC, so, it is more likely that PTEN is one of the important parameters which determine response to this therapeutic monoclonal antibody (Chen et al., 2015). Contrary to our results Karapetis et al., 2013, reported that in chemotherapy-refractory CRC, PTEN expression was neither prognostic nor predictive of benefit from cetuximab-based therapy.

The divergence of the results may be due to different methods of assessment and evaluation between tissue microarray and within cores. In addition, Loupakis et al., (2019) observed that the predictive value of PTEN was noticed only when the evaluated tumor tissue came from a metastatic tumor but not from the primary site.

Notably, loss of P21 is associated with better response to cetuximab based therapy while overexpression is associated with resistance to it, which were demonstrated in many previous studies (Ogino et al., 2005, Spano et al., 2008, and Jeffrey et al., 2010), which was inconsistent with our results.

Analysis of cell cycle progression in malignant cells gives a rationale for cetuximab and P21functional effects, pointing to the underlying mechanism of action (Terzuoli et al., 2017). Cetuximab has an important role in the induction of growth arrest at the G2/M phase. The increasing number of cells at $\mathrm{G} 2 / \mathrm{M}$ phase was related to induction of the CDK inhibitors; p21 and p27 through the silence of cyclin E/CDK2 which is the G1/S-promoting kinases that lead to arrest G1 arrest. G1/S checkpoint, which is controlled by two mechanisms related to $\mathrm{p} 21$ and mostly deregulated in CRC cells. Reduced the expression of $\operatorname{cyclin} B$ in cetuximab treated cells, leads to an adequate blocking of the G2/M and prevents cells from initiation of mitosis (Abbas et al., 2009, Kastan et al., 2004). Also, EGF/EGFR system impairment occurred by the cetuximab was found to be related to the ability to initiate a growth arrest in phases of G2/M in CRC cells that subsequently lead to a decrease in P21 (Li X et al..2010).

In this study, we also showed that there is an inverse association between $P 21$ expression and $O A R$ to cetuximab in mCRC, and both act in CRC cells by two related mechanisms. First, they could accelerate EGFR turnover by ubiquitination, and decrease EGFR receptor density, allowing less concentration of cetuximab to exert cancer inhibitory effects. Second, they lead to arrest in the cell cycle at the $\mathrm{G} 2 / \mathrm{M}$ phase by increasing expression of the $C D K$ inhibitors $p 21$ and $p 27$ to increase the apoptotic process (Huang et al., 2017, Coccia et al., 2016, and Kim et al., 2016).

\section{limitations}

The small sample size represented the main limitation. Moreover, the cause of death was not analyzed if it is therapy-related or disease-related. Further validation by using a large cohort of patients is needed.

In Conclusion, cetuximab proved efficacy in management of mCRC but it was found that not all patients respond to such therapy. Due to its high cost, it is better to identify markers that could be able to predict the response before starting therapy. As the IHC is a cheap and easy method of tissue protein markers assessment, we used such technique to detect the predictive roles of three biomarkers which are Amphiregulin, PTEN and $\mathrm{P} 21$. Our study revealed that high expression of both Amphiregulin, PTEN in addition to $P 21$ down regulation were associated with better response and improved outcome to cetuximab-based therapy which suggest that assessment of tissue protein expression of those marker might be beneficial in the therapy selection.

\section{Author Contribution Statement}

None.

\section{Acknowledgments}

We thank to our colleagues in pathology, medical and oncology departments in faculty of medicine Zagazig university for support in this work.

\section{Conflict of interest}

The authors clarify that there is no conflict of interest belonged to this research.

\section{References}

Abbas T, Dutta A (2009). p21 in cancer: intricate networks and multiple activities. Nat Rev Cancer, 9, 400-14.

Amin MB, Greene FL, Edge SB, et al (2017). Jeffrey E. Gershenwald; Robert K. Brookland. The Eighth Edition AJCC Cancer Staging Manual: Continuing to Build a Bridge From a Population-Basedto a More "Personalized" Approach to Cancer Staging. CA Cancer J Clin, 67, 93-99.

Chen Y, Shi Y, Lin J, et al (2015). Combined analysis of EGFR and PTEN status in patients with KRAS wild-type metastatic colorectal cancer. Medicine (Baltimore), 94, e1698.

Coccia A, Mosca L, Puca R, et al (2016). Extra-virgin olive oil phenols block cell cycle progression and modulate chemotherapeutic toxicity in bladder cancer cells. Oncol Rep, 36, 3095-3104.

Douillard JY, Siena S, Cassidy J, et al (2010). Randomized, phase III trial of panitumumab with infusional fluorouracil, leucovorin, and oxaliplatin (FOLFOX4) versus FOLFOX4 alone as first-line treatment in patients with previously untreated metastatic colorectal cancer: the PRIME study.

Asian Pacific Journal of Cancer Prevention, Vol 221033 
J Clin Oncol, 28, 4697-4705.

Ford KS, Garrett CR, Meropol NJ, et al (2007). Expression of epiregulin and amphiregulin and K-ras mutation status predict disease control in metastatic colorectal cancer patients treated with cetuximab. J Clin Oncol, 25, 3230-7.

Frattini M, Saletti P, Romagnani E, et al (2007). PTEN loss of expression predicts cetuximab efficacy in metastatic colorectal cancer patients. Br J Cancer, 97, 1139-45.

Hsu SM, Raine L, Fanger H (1981). Use of avidin-biotinperoxidase complex $(\mathrm{ABC})$ in immunoperoxidase techniques: a comparison between $\mathrm{ABC}$ and unlabeled antibody (PAP) procedures. J Histochem Cytochem, 29, 577-80.

Huang YF, Zhu DJ, Chen XW, et al (2017). Curcumin enhances the effects of irinotecan on colorectal cancer cells through the generation of reactive oxygen species and activation of the endoplasmic reticulum stress pathway. Oncotarget, 8 , 40264-75.

Jacobs B, DeRoock W, Piessevaux H, et al (2009). Amphiregulin and epiregulin mRNA expression in primary tumors predicts outcome in metastatic colorectal cancer treated with cetuximab. J Clin Oncol, 27, 5068-74.

Jeffrey S, Jorge Torres M, Nikhil W, et al (2010). Biomarker-based prediction of response to therapy for colorectal cancer current perspective. Am J Clin Pathol, 134, 478-90.

Karapetis CS, Jonker D, Daneshmand M, et al (2014). PIK3CA, BRAF, and PTEN status and benefit from cetuximab in the treatment of advanced colorectal cancer--results from NCIC CTG/AGITG CO.17. Clin Cancer Res, 20, 744-53.

Kastan MB, Bartek J (2004). Cell-cycle checkpoints and cancer. Nature, 432, 316-23.

Khelwatty S, Essapen SH, Bagwan I, et al (2017). Impact of coexpression of wild-type EGFR and its ligands determined by immunohistochemistry for response to treatment with cetuximab in patients with metastatic colorectal cancer Oncotarget, 8, 7666-77.

Khelwatty SA, Essapen S, Seddon AM, et al (2013). Prognostic significance and targeting of HER family in colorectal cancer. Front Biosci, 18, 394-421.

Kim E, Davidson LA, Zoh RS, et al (2016). Rapidly cycling Lgr5+ stem cells are exquisitely sensitive to extrinsic dietary factors that modulate colon cancer risk. Cell Death Dis, 7, e2460.

Li X, Fan Z, Ross S, et al (2010). Biomarker-based prediction of response to therapy for colorectal cancer current perspective Am J Clin Pathol, 134, 478-90.

Li XD, Miao SY, Wangb GL, et al (2010). Amphiregulin and Epiregulin expression in colorectal carcinoma and the correlation with clinicopathological characteristics. Onkologie, 33, 353-8.

Loupakis F, Pollina L, Stasi I, et al (2009). PTEN expression and KRAS mutations on primary tumors and metastases in the prediction of benefit from cetuximab plus irinotecan for patients with metastatic colorectal cancer. J Clin Oncol, 27, 2622-9.

Ma L, de Roquancourt A, Bertheau P, et al (2001). Expression of amphiregulin andepidermal growth factor receptor in human breast cancer: analysis of autocriny and stromal epithelial interactions. J Pathol, 194, 413-9.

Mao C, Liao RY, Chen Q (2010). Loss of PTEN expression predicts resistance to EGFR targeted monoclonal antibodies in patients with metastatic colorectal cancer. Br J Cancer, 102, 940 .

Mellinghoff IK, Cloughesy TF, Mischel PS (2007). PTEN mediated resistance to Epidermal Growth Factor Receptor Kinase inhibitors. Clin Cancer Res, 13, 370-82.

Negri, C Bozzetti, Lagrasta, P, et al (2010). PTEN status in advanced colorectal cancer treated with cetuximab. $\mathrm{Br} J$ Cancer, 102, 162 - 4.

Ogino S, Meyerhardt JA, Cantor M, et al (2005). Molecular alterations in tumors and response to combination chemotherapy with gefitinib for advanced colorectal cancer. Clin Cancer Res, 1, 6650-6.

Okada, Miyamoto H, Goji T, et al (2014). Biomarkers for predicting the efficacy of anti-epidermal growth factor receptor antibody in the treatment of colorectal cancer. Digestion, 89, 18-23.

Oliveras-Ferraros, S Cufi', B Queralt, et al (2012) Cross-suppression of EGFR ligands amphiregulin and epiregulin and de-repression of FGFR3 signalling contribute to cetuximab resistance in wild-type KRAS tumour cells. Br J Cancer, 106, 1406-14.

Razis E, Briasou lis E, Vrettou E, et al (2008). Potential value of PTEN in predicting cetuximab response in colorectal cancer: An exploratory study. BMC Cancer, 8, 234.

Sahin IH, Christopher RG (2014). Predicting cetuximab efficacy in patients with advanced colorectal cancer. Curr Biomarker Find, 4, 61-68.

Shao Y, Shen Y, Chen T, et al (2016). The functions and clinical applications of tumor-derived exosomes. Oncotarget, 7, 60736-75.

Siegel RL, Miller KD, Jemal A (2019). Cancer statistics. $C A$ Cancer J Clin, 69, 4-7.

Sood A, McClain D, Maitra R, et al (2012). PTEN gene expression and mutations in the PIK3CA gene as predictors of clinical benefit to anti-epidermal growth factor receptor antibody therapy in patients with KRAS wild-type metastatic colorectal cancer. Clin Colorectal Cancer, 11, 143-50.

Spano M, St'ephane S, Vignot F, et al (2008). Potential predictive markers of response to EGFR-targeted therapies in colorectal cancer. Crit Rev Oncol Hematol, 66, 21-30.

Sunakawa, Yang D, Moran M, et al (2016). Combined assessment of EGFR-related molecules to predict outcome of 1st-line cetuximab containing chemotherapy for metastatic colorectal cancer. Cancer Biol Ther, 7, 751-9.

Terzuoli E, Nannelli G, Frosini M, et al (2017). Inhibition of cell cycle progression by the hydroxytyrosol- cetuximab combination yields enhanced chemotherapeutic efficacy in colon cancer cells. Oncotarget, 8, 83207-24.

Ueno H, Kajiwara Y, Shimazaki H, et al (2012). New criteria for histologic grading of colorectal cancer. Am J Surg Pathol, 36, 193-201.

Yarden Y, Sliwkowski MX (2001). Untangling the ErbB signalling network. Nat Rev Mol Cell Biol, 2, 127-37.

Yonesaka K, Takegawa N, Satoh T, et al (2015). Combined analysis of plasma amphiregulin and heregulin predicts response to cetuximab in metastatic colorectal cancer. $P L O S$ One, 10, e0143132.

Yonesaka K, Zejnullahu K, Okamoto I, et al (2011). Activation of ERBB2 signaling causes resistance to the EGFR-directed therapeutic antibody cetuximab. Sci Transl Med, 3, 99ra86.

Zarkavelis G, Boussios S, Papadaki A, et al (2017). Current and future biomarkers in colorectal cancer. Ann Gastroenterol, 30, 613-21.

Zhang Y, Qu, X, Che X, et al (2018). Exosomes promote cetuximab resistance via the PTEN/Akt pathway in colon cancer cells. Braz J Med Biol Res, 51, e647.

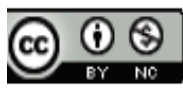

This work is licensed under a Creative Commons AttributionNon Commercial 4.0 International License. 